\title{
On apparent misalignment of collinear edges and boundaries
}

\author{
R. H. DAY \\ La Trobe University, Bundoora, Victoria, Australia \\ and \\ ANDREA PARKER HALFORD \\ Deakin University, Burwood, Victoria, Australia
}

\begin{abstract}
In two experiments, subjects adjusted various pairings of the top and bottom boundaries of two obliquely oriented outline bars (Experiment 1) and those of two similarly oriented complete and incomplete squares (Experiment 2) to apparent alignment. The data from the first experiment showed that the misalignment effects were determined jointly by the directional properties of the bar ends (vertical, oblique, and semicircular) and the pair of boundaries that were aligned (both top boundaries, top of upper bar with bottom of lower bar, bottom of upper bar with top of lower bar). The results from the second experiment showed that the misalignment effects were the same for the oblique boundaries of solid and outline squares and persisted when the squares were reduced to two parallel lines. The effect was undiminished when the ends of the parallels were aligned, but was markedly reduced when pairs of parallels themselves were aligned. The outcomes of the two experiments are explained in terms of the apparent positions of the oblique boundaries. It is proposed that these vary with the positions of the elements (bar or square) relative to the visual field, the position of the boundaries relative to the stimulus elements, and the positions of the boundaries relative to axes that are delineated by the parallel adjacent ends of bars and sides of squares. This relative-position basis for apparent misalignment is held to be the basis of misalignment effects in other figures.
\end{abstract}

In a previous series of experiments (Day, Stecher, \& Parker, 1993), it was shown that the collinear edges of oblique bars with rounded ends appear to be slightly but reliably misaligned. This effect was nearly doubled when one of the bars was moved vertically in the course of adjusting it to apparent alignment with the other. When (as in Figure 1A) the ends of the bars and movements of one bar during adjustment were both vertical, the misalignment effect was about six times greater. However, it was reduced when the ends of the bars remained vertical while adjustment movements were orthogonal to the axis of the oblique bars (as is shown in Figure 1B). In the last of the four experiments, it was found that although the orientation and form of the inner pair of bar ends had a marked effect on apparent misalignment, those of the outer pair had no effect at all. Finally, when the inner ends of the bars abutted on vertical parallel lines, as in the conventional Poggendorff figure, the misalignment effect was about the same as that with vertical bar ends. This suggested that vertical ends and vertical parallels

This research was supported by a grant to the first author by the Australian Research Council. The assistance of Vladimir Kohout, Rosemary Williams, and Mike Durham, in photography, preparation of figures, and computer programming, respectively, is gratefully acknowledged. Address correspondence to R. H. Day, La Trobe University, Department of Psychology, Bundoora, Victoria 3083, Australia. are more or less equivalent in determining apparent misalignment of edges.

In the course of these experiments, a misalignment effect involving the collinear opposite edges of oblique bars was noted. When the top edge of the uppermost oblique bar was collinear with the bottom edge of the lowermost bar, the two edges appeared to be grossly misaligned; the top edge appeared far too high for alignment with the bottom edge, as is shown in Figure 1B. However, when this relationship was reversed, so that the bottom edge of the uppermost bar was aligned with the top edge of the lowermost bar, the two edges appeared to be slightly misaligned or more or less aligned, as is shown in Figure 1C. Subsequent informal observations showed that these strong and weak effects also occurred with the boundaries of outline bars and squares.

Almost all of the earlier investigations of apparent misalignment of collinear features have involved straight lines, usually in the Poggendorff figure or its numerous variants (see Hotopf \& Hibberd, 1989). For this reason, the effects in Figure 1, involving the same and opposite edges of bar elements, have not previously been described or studied. The previous experiments with bars (Day \& Stecher, 1992; Day et al., 1993) showed that the orientation of the ends of the bars are also strongly implicated in apparent misalignment. Therefore, in the first of the two present experiments, three pairings of the boundaries of outline bars and three forms of bar ends 
A

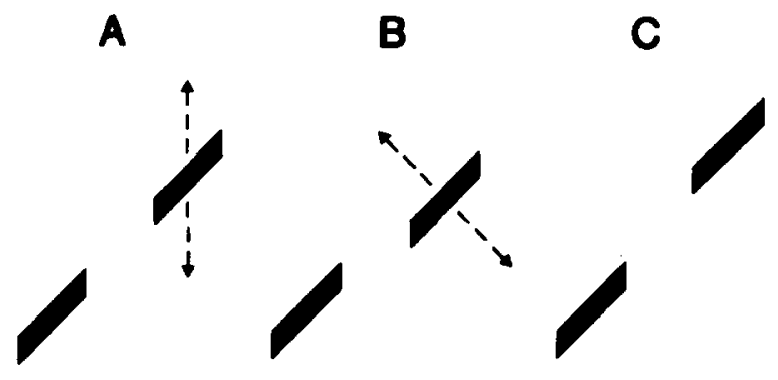

Figure 1. Apparent misalignment of the collinear edges of solid bars. The effect is clearty evident when the top edge of the upper bar is aligned with the top edge of the lower $\operatorname{bar}(A)$, greater when the top edge of the upper bar is aligned with the bottom edge of the lower bar $(B)$, and is much smaller when the bottom edge of the upper bar is aligned with the top edge of the lower bar (C). The dotted lines refer to the direction of movement of the adjustable bar in the experiments reported here (A) and in some earlier experiments (B).

were systematically combined in nine conditions. In the second experiment, squares were combined as in Figures $1 \mathrm{~B}$ and $1 \mathrm{C}$. The purpose of this experiment was to ascertain whether the effects for the different conditions of boundary alignment occur with elements other than bars, and whether they vary between solid and outline elements and between complete and incomplete elements.

It can be pointed out at this stage that the outcomes of the two experiments have led to an explanation of apparent misalignment of edges and boundaries in terms of their apparent positions. It is argued that the apparent positions of the aligned boundaries and edges are determined by the positions of the two stimulus elements in the visual field-- those of the aligned features relative to the elements and those of the elements relative to the axes that are delineated by the inner ends of the bars or the inner sides of the squares.

\section{EXPERIMENT 1}

The purpose of Experiment 1 was to ascertain whether the form of the bar ends combines with the pair of boundaries to be aligned to determine the extent of apparent misalignment. The bar ends were vertical (V), semicircular $(\mathrm{N})$, and oblique and orthogonal to the axis of the bars $(\mathrm{O})$. The boundary alignment conditions were the top boundary of the upper bar aligned with the top boundary of the lower bar (TT), the top of the upper bar with the bottom of the lower bar (TB), and the bottom of the upper bar with the top of the lower bar (BT). ${ }^{1}$ Thus, there were nine stimulus conditions: V-TT, V-TB, V-BT, N-TT, N-TB, N-BT, O-TT, O-TB, and O-BT. These are shown in Figure 2. Given the results from the earlier experiments (Day et al., 1993) and the informal observations of the arrangements in Figure 1, it was expected that, of the three delineation conditions, Condition $\mathrm{V}$ would give rise to the greatest misalignments, and of the three boundary alignment conditions, Condition TB would do so.

\section{Method}

Subjects. Twelve subjects, 6 women and 6 men, were paid for their participation.

Apparatus. The apparatus is described in Day and Stecher (1992) and Day et al. (1993). The stimulus figures were presented symmetrically around the center of a $27.5 \times 20.4 \mathrm{~cm}$ screen of a personal computer and viewed from a distance of approximately $114 \mathrm{~cm}$ ( $10 \mathrm{~mm}$ at the screen subtended $30^{\prime}$ at the eye). The computer generated the figures, presented them in a different random order for each subject, varied the starting position of the adjustable bar on the right, and recorded, averaged, and stored individual scores. The adjustable bar could be moved obliquely at right angles to the bars (see Figure 2) by pressing one of two directionally marked keys, one for upward movement and the other for downward movement. When the keys were held down, the bar moved continuously and smoothly; when tapped, it moved stepwise in .37-mm steps. Pressing a third key brought up the next figure in the randomized stimulus sequence.

Stimulus conditions. Each figure consisted of two $45^{\circ}$ oblique outline bars, $40 \mathrm{~mm}$ long, $8 \mathrm{~mm}$ wide, and separated by $40 \mathrm{~mm}$ when exactly aligned (see Figure 2 ). The lines forming the bound-

\section{$V-T T$ \\ $\overline{\mathrm{x}}=\mathbf{3 . 7 8}$ \\ (0.52)}

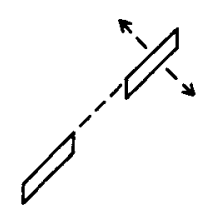

$N-T T$

$\overline{\mathrm{x}}=1.17$

(0.35)

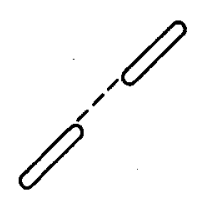

$$
\begin{gathered}
0-T T \\
\bar{x}=1.27 \\
(0.35)
\end{gathered}
$$

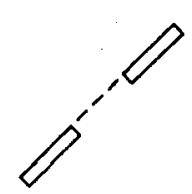

Figure 2. The nine stimulus conditions of Experiment 1. The ends of the bars were vertical (V), semicircular (N), and oblique and at right angles to the edges (O). The aligned edges (dotted lines) were the top of the upper bar with the top of the lower bar (TT), the top of the upper bar with the bottom of the lower bar (TB), and the bottom of the upper bar with the top of the lower bar (BT). The mean misalignment effects (in millimeters) and their standard errors (in parentheses) are also shown. 
aries of the bars were $0.8 \mathrm{~mm}$ thick with a luminance of about $9 \mathrm{~cd} / \mathrm{cm}^{2}$. Their ends were vertical, semicircular, or oblique at $45^{\circ}$ to the axis of the bars, as shown in Figure 2. The dotted lines in the figure indicate the boundaries to be aligned.

Procedure. The subjects' task was to adjust the oblique bar on the right, using the two keys, so that the two nominated boundaries appeared to be perfectly aligned. The nine stimulus conditions were presented in a different random order to each subject. Four adjustments to apparent alignment were made for each condition-two from an initial position approximately $18 \mathrm{~mm}$ below the point of true alignment and two from a position the same distance above it. These starting positions alternated over the four adjustments. All four adjustments for one condition were completed before the next condition was introduced. The subjects were permitted to hold down the relevant key for continuous movement of the bar, tap it for stepwise movements, or use both modes, as they preferred. There was no time limit for the adjustment. The subjects were told before each adjustment which boundaries to align, and this directive was also indicated in the top left corner of the screen as top, top bottom, or bottom top. Practice trials were run before the experimental trials were introduced.

Scoring. The score for each condition was always the mean vertical difference between true and apparent alignment, in millimeters, for the four adjustments. For example, in Figure 1A, the top and bottom edges of the bar on the right appear too high for alignment with the collinear edges of the bar on the left, so the bar on the right would have to be moved downward for the edges to appear aligned. For all of the figures, this direction of adjustment was scored as positive, and the opposite direction was scored as negative.

\section{Results and Discussion}

The mean misalignment scores and their standard errors (in parentheses) are also shown in Figure 2. In the interest of clarity and in order to show the interrelationships between the mean misalignments for the nine conditions, the means and standard errors are also plotted as histograms in Figure 3, with the standard errors represented by vertical "Ts" at the top of the bars. It can be seen from this figure that the means for the vertical bar ends (V) were consistently greater than those for semicircular $(\mathrm{N})$ and oblique $(\mathrm{O})$ ends across the three boundary alignment conditions, and that those for Condition TB were consistently greater than those for Conditions TT and BT across the three bar end conditions. It can also be noted that the greatest effect occurred for Condition V-TB and the smallest for Condition O-BT. Separate $t$ tests showed that the means for eight of the nine stimulus conditions were significantly greater than zero, at $p<.02$ or better, and one (for O-BT) was not $(p>.05)$. A two-way analysis of variance (ANOVA), with direction of the bar ends (D) and the boundaries that were aligned $(B)$ as main factors showed that both factors were significant $[F(2,22)=17.16, p<.0001$, and $F(2,22)=14.13, p<.0001$, respectively]. The interaction between the two factors was also significant $[F(4,44)=$ $14.13, p<.001]$. A more detailed analysis of the differences across the three forms of bar ends and the three boundary alignment conditions was made by using the Newman-Keuls procedure. This showed that the three means for Condition $\mathrm{V}$ were significantly different from each other at $p<.05$ or better. Of the three means for Condition N, that for Condition TB was significantly different from those for Conditions TT and BT $(p<.01)$, which were not different from each other, and the three means for Condition $\mathrm{O}$ were also significantly different from each other $(p<.02)$. Of the three means for Condition TT, that for Condition V was significantly greater than those for Conditions $\mathrm{N}$ and $\mathrm{O}(p<.01)$, which were not different from each other. For Condition TB, the mean for Condition $V$ was significantly greater than those for Conditions $\mathrm{N}$ and $\mathrm{O}(p<.01)$, which were not significantly different from each other. For Condition BT, the means for Conditions $\mathrm{V}$ and $\mathrm{N}$ were not significantly different from each other, and both were significantly greater than that for Condition $\mathrm{O}(p<.05)$.

The outcome of this experiment confirms the earlier finding (Day \& Stecher, 1992; Day et al., 1993) that the form of the ends of the bars is a major determinant of apparent misalignment of collinear boundaries. In addition, the results show that the positions in the bars of the aligned boundaries--whether they are topmost in both bars, or topmost in one bar and bottommost in the otheris also a major factor. In short, apparent misalignment of boundaries was jointly determined by the form of the bar ends and the positions of the boundaries relative to the bars. These data have suggested a basis for apparent misalignment of boundaries and edges in terms of the relative positions of the boundaries. This is discussed further in the General Discussion.

\section{EXPERIMENT 2}

Three considerations led to the second experiment. First, the presence and absence of apparent misalignment of edges and boundaries has been demonstrated only with two-bar figures, such as those in Figure 1.

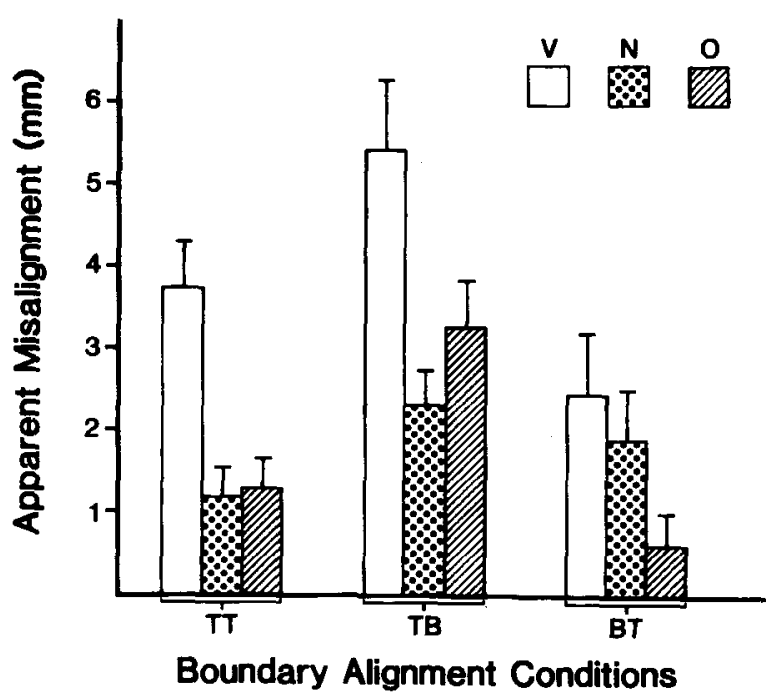

Figure 3. Comparison of apparent misalignment (in millimeters) and standard errors for the nine conditions (see Figure 2) of Experiment 1. Ends of bars were vertical ( $V$ ), semicircular $(N)$, and oblique $(O)$. Boundary alignments were tops of upper and lower bars (TT), top of upper bar with bottom of lower bar (TB), and bottom of upper bar with top of lower bar (BT). 
Among the possible explanations for apparent misalignment is a change in the apparent orientation of the bars in the direction of either the vertical or horizontal axis. Such "normalization" with elongated stimulus elements has been claimed as a component of apparent misalignment in other figures (see Hotopf \& Hibberd, 1989). Although such normalization would seem to be unlikely for bars aligned in the $45^{\circ}$ axis (i.e., halfway between the vertical and horizontal), it was nevertheless considered prudent to investigate the possibility. Square elements with their corners in the vertical and horizontal axes were used, and the features to be aligned were at $45^{\circ}$, as were the bars (see Figure 4 ). With such an arrangement, a change in apparent orientation due to normalization would be highly unlikely.

The second consideration concerned the equivalence, or otherwise, of outline and solid (i.e., "filled-in") stimulus elements. Although the misalignment effects with these two classes of elements appeared, on casual inspection, to be about the same, this had not been formally tested. The main reason for doing so was the possibility that the boundaries of outline figures might be more easily perceptually "isolated" and aligned in the same axis than would the edges of solid figures. This possible difference would presumably lead to misalignment effects that were smaller with boundaries than with edges.

Third, we asked whether apparent misalignment occurs with the boundaries of incomplete figures as well. An answer to this question bears on the relationship between the effects described here and those associated with the arc and chevron figures reported by Day, Jee, and Duffy (1989). It was found that apparent misalignment of points in a horizontal axis is markedly reduced when these figures are incomplete.

These issues were pursued in Experiment 2 by using the pairs of complete and incomplete squares with oblique edges or boundaries, as is shown in Figure 4. In Figures $4 \mathrm{~A}, 4 \mathrm{C}, 4 \mathrm{E}$, and $4 \mathrm{G}$, the bottom oblique feature-edge, boundary line, or pair of line ends - of the upper element on the right was aligned with the top feature of the lower element on the left, as for Condition BT in Experiment 1. In Figures 4B, 4D, 4F, and 4H, the top feature of the upper element on the right was aligned with the bottom feature of the lower element on the left, as for Condition TB in Experiment 1. The square elements in Figures $4 \mathrm{~A}$ and $4 \mathrm{~B}$ were solid. In Figures $4 \mathrm{C}$ and $4 \mathrm{D}$ they were completely outlined, and in Figures $4 \mathrm{E}, 4 \mathrm{~F}$, $4 \mathrm{G}$, and $4 \mathrm{H}$ they were incompletely outlined. Two boundary lines were aligned in Figures $4 \mathrm{E}$ and $4 \mathrm{~F}$, and two pairs of line ends were aligned in Figures $4 \mathrm{G}$ and $4 \mathrm{H}$.

\section{Method}

Subjects. There were 12 new subjects, 6 women and 6 men, drawn from the same source as that used for Experiment 1 . They were paid for their participation.

Apparatus and Procedure. The apparatus, procedure, and scoring were the same in all respects as those in Experiment 1.

Stimulus conditions. The solid, complete-outline, and incompleteoutline elements in Figure 4 were $40 \mathrm{~mm}$ square, with their aligned
A

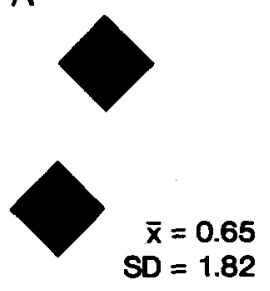

C
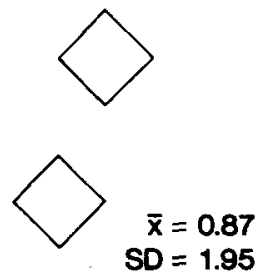

$\mathbf{E}$

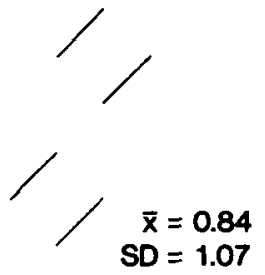

G

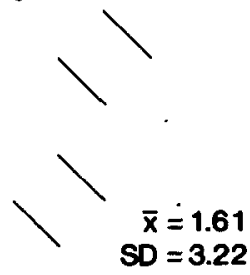

B

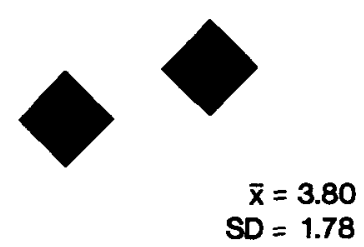

D

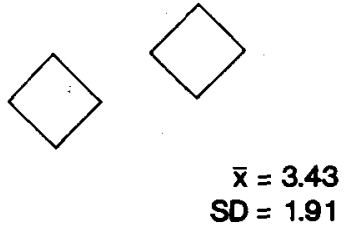

$\mathbf{F}$

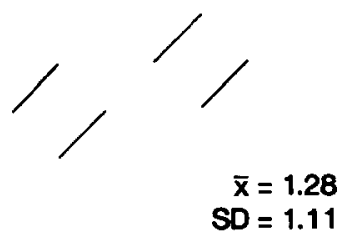

H

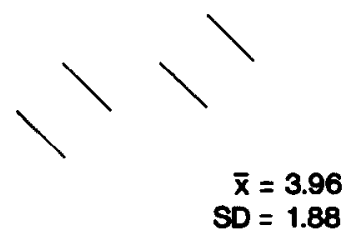

Figure 4. The eight stimulus conditions of Experiment 2, together with their mean misalignment effects (in millimeters) and standard deviations $(S D)$. The figures included solid $(A$ and $B)$ and outline (C and $D$ ) squares, and outline-incomplete ( $E$ and $F, G$ and $H$ ) squares with two edges or boundaries obliquely aligned $(E$ and $F$ ) and two pairs of line ends aligned ( $G$ and $H)$.

features at $45^{\circ}$ and separated by $40 \mathrm{~mm}$ when exactly aligned. The element on the right again moves obliquely at right angles to the oblique boundaries for adjustments to alignment.

\section{Results and Discussion}

The mean misalignments are shown with their standard deviations in Figure 4. It can be seen that the means for Condition BT were consistently smaller than those 
with the same pair of elements for Condition TB. Note that for Condition BT, three means (A, C, and E) were relatively small, and one $(\mathrm{G})$ was larger. For Condition $\mathrm{TB}$, three means $(\mathrm{B}, \mathrm{D}$, and $\mathrm{H})$ were relatively large, and one $(F)$ was smaller.

Separate $t$ tests showed that, of the four means for Condition BT, one (E) was significant, and three $(\mathrm{A}, \mathrm{C}$, and $\mathrm{G})$ were not $[t(11)=2.72, p<.02 ; t(11)=1.24, p>$ $.05 ; t(11)=1.55, p>.05$; and $t(11)=1.78, p>.05$, respectively]. The same tests for Condition TB showed that all four means were significant [for $\mathrm{B}, t(11)=7.40$, $p<.001 ; \mathrm{D}, t(11)=6.22, p<.001 ; \mathrm{F}, t(11)=3.99, p<$ $.01 ; \mathrm{H}, t(11)=7.30, p<.001]$. A two-way ANOVA, with type of stimulus element $(\mathrm{E})$ and the positions (TB or BT) of features to be aligned (A) as main factors showed that both factors as well as the interaction between them were significant [for $\mathrm{E}, F(3,11)=5.47, p<.01$; for A, $F(1,11)=16.37, p<.001$; and for their interaction, $F(3,33)=4.47, p<.01]$. Separate ANOVAs were carried out for the two alignment conditions, BT and TB. These showed that the type of stimulus element was a significant factor for Condition TB $[F(3,11)=14.03$, $p<.001]$, but it was not for Condition BT $[F(3,11)=$ $0.76, p>.50]$. Newman-Keuls tests of the differences between the means for Condition TB showed that the mean for Figure $4 \mathrm{~F}$ was significantly smaller than those for Figures $4 \mathrm{D}, 4 \mathrm{~B}$, and $4 \mathrm{H}$, and that these three means were not different from each other.

These data provide clear answers to the three questions posed in the introduction. First, the effects that had been previously found for bars under Conditions BT and TB were essentially the same for stimulus figures consisting of squares, in which normalization toward the main axes of space would be very unlikely. Second, the effects occurred as strongly under Condition TB with solid squares as they did with outline squares, and they were absent for both solid and outline squares under Condition BT. Third, the effect with incomplete squares was as great as that with complete squares when the ends of the two pairs of parallel lines were aligned, but was markedly less when two lines flanked by parallel lines were aligned.

\section{GENERAL DISCUSSION}

The outcomes of the present two experiments confirm and extend those reported earlier on the involvement of bar ends in apparent misalignment of collinear bar edges and boundaries (Day \& Stecher, 1992; Day et al., 1993). In the first place, the results verify that the form of the bar ends is a critical determinant of apparent misalignment; vertical ends give rise to large effects, oblique orthogonal ends give rise to smaller or negligible effects, and semicircular ends give rise to effects of about the same size as those for orthogonal ends. Second, the positions of collinear boundaries in both bar and square elements have been shown to be closely involved and to interact with bar ends in deter- mining the size of the effect. Third, misalignment effects with incomplete squares are greater for the collinear ends of lines (Figures $4 \mathrm{G}$ and $4 \mathrm{H}$ ) than for the collinear lines flanked by parallel lines (Figures $4 \mathrm{E}$ and 4F).

The main issues for consideration are an explanation of these outcomes and a determination of whether apparent misalignment in other figures-in particular, the various forms of the Poggendorff figure-can be accounted for in essentially the same terms. In proposing an explanation, it is relevant to draw attention again to data from the earlier series of experiments (Day et al., 1993), since they bear closely on an explanation of those reported here.

The earlier results showed that apparent misalignment of bar edges with vertical inner bar ends and vertical parallels are more or less the same, that only the inner pair of bar ends, not the outer ends, are involved, and there is a small but reliable horizontal-vertical (HV) contribution to the misalignment effect with $45^{\circ}$ oblique bars. The first of these outcomes indicates that inner, parallel bar ends and parallel lines are functionally equivalent as determinants of apparent misalignment. The second outcome suggests that the inner parallel ends of bars and sides of squares are critically involved. The third outcome indicates that the HV spatial anisotropy contributes slightly to the misalignment effect. This finding is strongly supported by experiments with a pair of oblique lines (Day, 1973a) and the Poggendorff figure with vertical and horizontal parallels (Day \& Dickinson, 1976). It will be assumed henceforth that the HV component also contributed to the effects in Experiments 1 and 2 .

It is proposed that the misalignment effects in Figures 2 and 4 are mainly attributable to the apparent positions of the aligned features, and that these positions are determined singly or jointly by three sets of relationships in the stimulus array. The first involves the positions of the stimulus elements (bars or squares) relative to the rectangular field, the second to the positions of the aligned features (boundaries, edges, line ends) relative to the elements, and the third to positions of the aligned features relative to the inner pair of bar ends or sides of squares. The data from these and other experiments indicate that one or more of these relationships determine the apparent position of the bars and thereby their apparent misalignment. The three spatial relationships warrant further description.

In all of the conditions represented in Figures 2 and 4 , the stimulus elements were obliquely disposed in a $45^{\circ}$ axis. In consequence, the element on the right was always higher in the stimulus field than that on the left. Furthermore, the difference in elevation varied according to the pair of boundaries or edges that were aligned. The greatest difference occurred for Condition BT, the smallest for Condition TB, and that for Condition TT was intermediate. The aligned features also varied in position relative to the stimulus element; they were either 
uppermost or lowermost in the element. In addition, the aligned features varied relative to the axes that were delineated by the inner pair of bar ends or sides of squares. Relative to the vertical axes, the aligned features were differently positioned, with that on the right higher than that on the left, and relative to the oblique axis the features were identically positioned. In the case of the semicircular bar ends, no particular direction was delineated, so that position relative to an axis was not involved in determining apparent positions.

The contributions of these relative positions can be illustrated by reference to Conditions V-TB and O-BT in Figure 2, for which the misalignment effects were, respectively, greatest $(5.87 \mathrm{~mm})$ and negligible $(0.58 \mathrm{~mm})$. For Condition V-TB, the bar on the left is higher in the field than that on the left, uppermost rather than lowermost in the bar, and higher relative to the vertical axis. In Condition O-BT, the boundary on the right is still higher in the field, is lowermost rather than uppermost in the bar, and is in the same position relative to the oblique axis.

The same reasoning can be extended to the other conditions of Experiment 1 and to those of Experiment 2. For example, in Conditions V-BT and V-TB (Figure 2), the elements on the right are both higher in the field than those on the left, and the boundaries on the right are higher, relative to the vertical, to the same extent. However, the same boundaries are lowermost in the element, whereas those on the left are uppermost. The consistently greater effects for the vertical conditions than for the semicircular and oblique conditions across all boundary alignments can be attributed to the higher positions of the right boundary than those of the left relative to the vertical. Similarly, the consistently greater effects for Condition TB than for Conditions TT and BT across all bar end conditions in Figure 2 can be attributed to the uppermost position of the aligned boundary on the right relative to that on the left.

It is to be noted that although no direction in particular was delineated by the inner semicircular ends in Condition $\mathrm{N}$, movements of the bar on the right during adjustments to apparent alignment did so. As pointed out previously, it was shown by Day et al. (1993) that the effectiveness of delineation of direction by a bar end is enhanced or reduced according to whether the direction of delineation by bar movement is the same or different. It can be presumed that the relatively large and significant misalignment effect in Condition N-BT and the negligible effect in Condition O-BT were due in part to the more effective delineation of the oblique direction by both of these factors in the latter condition.

The results for Experiment 2 can be accounted for in the same terms. In this experiment, the inner parallel sides of the square element were orthogonal to the aligned features for all conditions shown in Figure 4. That is to say, the aligned features were not misaligned relative to the axes delineated by the inner parallel sides of the square and by the movement of the adjustable square. It is to be noted that, like the result for Condition O-BT in Figure 2, three results for the equivalent condition in Figure 4 (Figures $4 \mathrm{~A}, 4 \mathrm{C}$, and $4 \mathrm{G}$ ) were negligible, and one (Figure $4 \mathrm{E}$ ) was slight but significant.

The two issues remaining for consideration are the marked difference in apparent misalignment between the conditions with incomplete elements in Experiment 2 and a determination of whether this apparent-position explanation can account for apparent misalignment in other figures.

In Figures $4 \mathrm{E}$ and $4 \mathrm{~F}$, a pair of oblique lines each flanked by a parallel line was aligned, and in Figures $4 \mathrm{G}$ and $4 \mathrm{H}$, two pairs of line ends were aligned. In the first case, the two lines were entirely separated from the parallels, so it is likely that they were no longer processed as the upper and lower boundaries of squares; but as separate entities. However, in Figures $4 \mathrm{G}$ and $4 \mathrm{H}$, the collinear pairs of line ends were inseparably the upper and lower extremities of pairs of line elements and were presumably processed as such. It is suggested, therefore, that the positions of the aligned features relative to the stimulus elements serve as a determinant of apparent position and, thereby, of apparent misalignment when the features are intrinsic to the elements, as with the boundaries of complete bars and squares and with the ends of lines.

In the conventional form of the Poggendorff figure and in its numerous variants, the collinear oblique lines
A
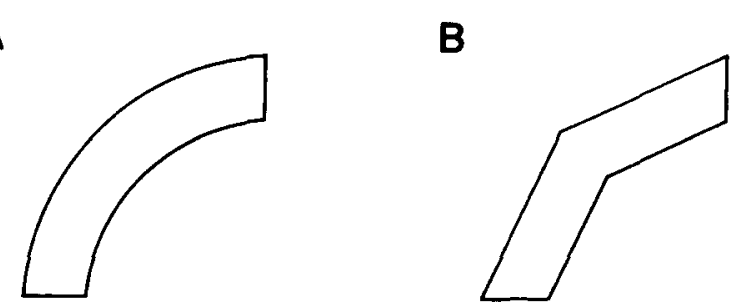

C

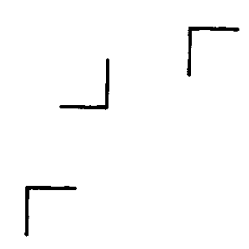

D

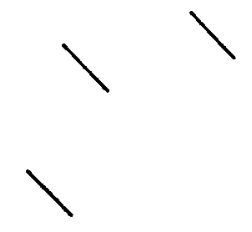

Figure 5. Apparent misalignment of collinear features in arc (A), chevron (B), Morinaga angle (C), and parallel-line (D) figures. In Figures $A$ and $B$, the outer ends of the top boundary and the center of the lower boundary are obliquely aligned, but appear to be misaligned; the outer ends appear to be located too obliquely upward for alignment. In Figures $C$ and $D$, the apexes and line ends are aligned, but appear to be misaligned; the outer apexes and line ends also appear to be located too obliquely upward for alignment. 


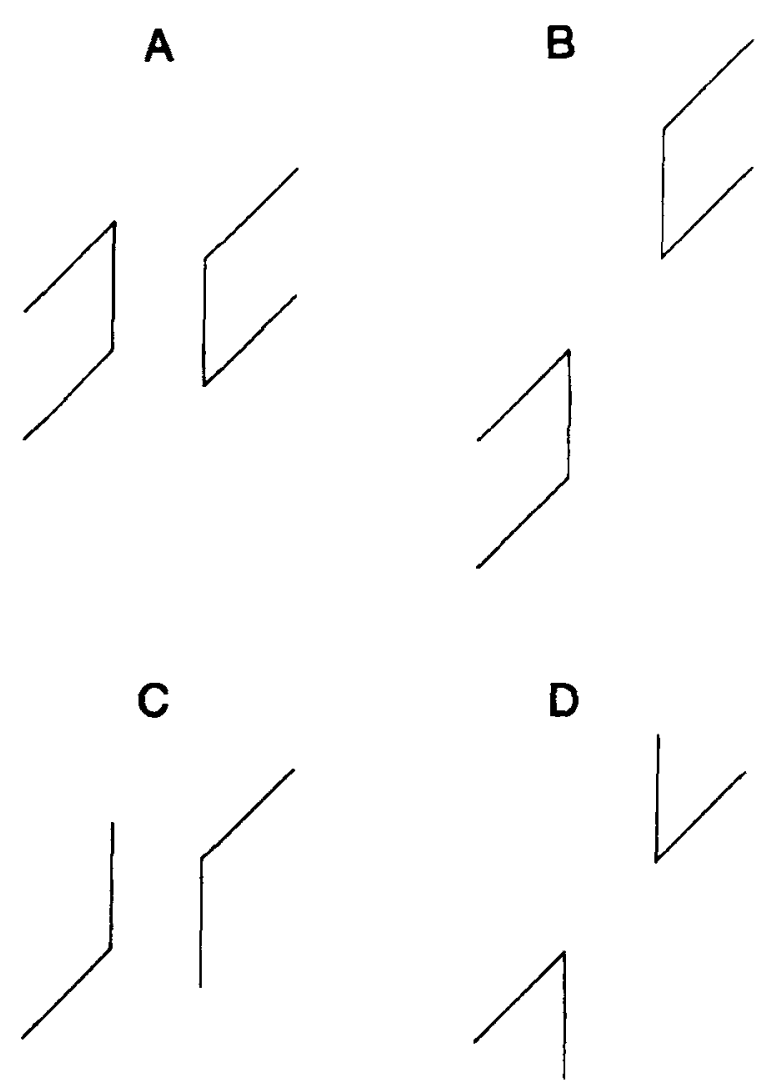

Figure 6. The obtuse- and acute-angle versions of the Poggendorff figure. Collinear obliques (C and D) are reduced forms of the bar figures with collinear boundaries ( $A$ and $B$ ).

are positioned one above the other relative to the vertical, which is delineated by the parallels (see Hotopf $\&$ Hibberd, 1989). In the arc and chevron figures described by Day et al. (1989) and shown in Figures 5A and 5B, the ends of the upper boundary (arc or obtuse angle) and the center of the lower boundary are obliquely aligned, but they appear to be misaligned. The ends of the upper boundary appear to be located too obliquely upward for alignment with the center of the lower boundary. In the two versions of the Morinaga figures, shown in Figures 5C (Morinaga \& Ikeda, 1965; Restle, 1976) and 5D (Day, Bellamy, \& Norman, 1983), the upper extremities (apexes and ends) of the outer elements (angles and lines) are obliquely aligned, but appear to be misaligned. The extremities of the outer elements appear to be located too obliquely upward for alignment with the center extremity. As is the case for the aligned boundaries and edges in Figure 1, it is proposed that the aligned features in the Poggendorff, arc and chevron, and the Morinaga figures are outcomes of their apparent positions. These in turn are held to be due to their positions relative to a defined axis, as in the Poggendorff figure; a stimulus element, as in the arc and chevron figures; and jointly to the positions of the elements in the visual field and those of the aligned features in the elements, as in the Morinaga figures.

Finally, the apparent-position explanation of apparent misalignment offers a solution to the long-standing puzzle of the Poggendorff effect when the conventional figure is reduced to obtuse- and acute-angle forms, as is shown in Figures 6C and 6D. In the obtuse-angle form, the misalignment effect is about the same as that in the conventional figure, and in the acute-angle form, it is slight or negligible (Day, 1973b; Green \& Hoyle, 1964; Pressey \& den Heyer, 1968; Restle, 1969). As can be seen in Figure 6, the obtuse- and acute-angle versions are essentially reduced versions of the bars in Conditions V-TB and O-BT, respectively, in Figure 2. The bars in Figures 6A and 6B have been reduced in Figures 6C and $6 \mathrm{D}$ to a vertical bar end and one oblique boundary, with the boundary uppermost in the former and lowermost in the latter.

In summary, apparent misalignment of collinear features in the present and previous experiments (Day \& Stecher, 1992; Day et al., 1993) is interpreted here in terms of the apparent positions of the features. The positions of features in a particular axis are held to undergo change in the direction of the positions of the elements relative to the visual field, relative to the elements, and relative to the axes that are delineated by the inner ends or sides of the elements. We also claim that a change in the apparent positions of collinear features is the basis of apparent misalignment in other stimulus figures as well, including the Poggendorff figure and its derivatives. The processes that are involved in the integration of relative-position information and weighted contributions of the three relativities that have been identified are issues for further inquiry.

\section{REFERENCES}

DAY, R. H. (1973a). The oblique line illusion: The Poggendorff effect without parallels. Quarterly Journal of Experimental Psychology, 25, 535-541.

DAY, R. H. (1973b). The Poggendorff illusion with obtuse and acute angles. Perception \& Psychophysics, 14, 590-596.

Day, R. H., Bellamy, S., \& Norman, A. (1983). On the Morinaga misalignment illusion. Journal of Experimental Psychology: Human Perception \& Performance, 9, 113-125.

DAY, R. H., \& DiCKInson, R. G. (1976). The components of the Poggendorff illusion. British Journal of Psychology, 67, 537-552.

DaY, R. H., JeE, F. M., \& DufFY, F. M. (1989). Visual misalignment in arc and chevron figures. Journal of Experimental Psychology, 15, 762-770.

DAY, R. H., \& STECHER, E. J. (1992). Some variant forms of the Poggendorff illusion and their implications for an explanation. Bulletin of the Psychonomic Society, 30, 26-28.

Day, R. H., Stecher, E. J., \& Parker, A. L. (1993). The effect of edge orientation and movement direction on the apparent misalignment of collinear bars. Perception \& Psychophysics, 53, 642-647.

Green, R. T., \& Hoyle, E. M. (1964). The influence of spatial orientation on the Poggendorff illusion. Acta Psychologica, 22, 348365 .

Hotopf, W. H. N., \& HibBERD, M. C. (1989). The role of angles in inducing misalignment in the Poggendorff figure. Quarterly Journal of Experimental Psychology, 41A, 355-383. 
Morinaga, S., \& Ikeda, H. (1965), Paradox in displacement in geometric illusion and the problem of dimensions: A contribution to the study of space perception. Japanese Journal of Psychology, 36, 231-238.

Pressey, A. W., \& den Heyer, K. (1968). Observations on Chiang's "new" theory of geometrical illusions. Perception \& Psychophysics, 4, 313-314.

Restle, F. (1969). Illusions of bent line. Perception \& Psychophysics, 5, 273-274.

Restle, F. (1976). Morinaga's paradox and figure-ground organization. Perception \& Psychophysics, 20, 153-156.

\section{NOTE}

1. A preliminary experiment with oblique-ended, filled-in bars, such as those in Figure 1, showed that there was no difference in apparent misalignment between the conditions in which the two top edges were aligned and those in which the two bottom edges were aligned. For this reason, and to reduce the number of alignment trials undertaken by the subjects, the latter condition was not included.

(Manuscript received January 13, 1993; revision accepted for publication April 21, 1994.) 